\title{
PROJECT OF A THREE REFLECTION TELESCOPE FOR WIDE FIELD ULTRAVIOLET OBSERVATIONS
}

\author{
A. AMORETTI, M. BADIALI, A. PREITE-MARTINEZ and R. VIOTTI \\ Istituto Astrofisica Spaziale, CNR, Via Fermi, 21, 00044 Frascati RM, Italy
}

\begin{abstract}
We present a project of a three-reflection $1.5 \mathrm{~m} \mathrm{f} / 3$ aplanatic wide-field telescope, in view of a small-satellite mission for the all-sky survey in the Ultraviolet.
\end{abstract}

\section{Wide Field Astronomy}

Of fundamental importance for our knowledge of the Universe is the exploration of the full sky in the whole electromagnetic spectrum. Complete sky surveys have been made in radio, infrared, optical and X-ray frequencies which have unveiled many unexpected phenomena, and have disclosed new categories of astrophysical objects, which have been later studied in more details with "pointed" experiments. But, as far as the Ultraviolet is concerned, no complete sky survey has been so far made, in spite of the important results obtained by the many UV space experiments in the past two decades, which have clearly indicated that a very large amount of astrophysical phenomena still remain to be discovered in the UV.

In order to perform the UV all-sky survey, a well corrected wide field telescope is required. The investigation should be done in different wavelength bands, typically three, in order to determine the gross energy distribution of each source, and the interstellar extinction. The FOV should be large enough (one to a few square degrees) to complete the survey in a reasonable time. With square fields of $90^{\prime} \times 90^{\prime}$, and with $10^{\prime}$ overlapping of adjacent fields, the whole sky can be imaged with some 23000 pointings, which should require a $2-3$ year mission. The experiment does not ask for a very high angular resolution, because this would bring the information to be stored, on-board analyzed, and transmitted to Earth to a level which can hardly be handled with the current techniques. A reasonable value for the resolution should be 1 arcsec. The main requirements of the telescope should be a FOV diameter of at least $2 \mathrm{deg}$, unvignetted, protected from straylights, with an image size of less than $1^{\prime \prime}$ in the whole FOV. The linear scale should be around 10 to $30 \mathrm{micron} /$ arcsec to be compatible with the currently used detectors. Clearly, very large detectors with a large number of elements (pixels) are needed.

Other important requirements should be the flatness of the surface of better image definition, the absence of refractive media, and the compactness and structural simplicity of the system. Ideal instruments capable to fullfill all these requirements are the three reflection telescopes described by Amoretti et al. (1989). In view of a small-satellite mission for the all-sky UV survey, we are considering telescopes of this kind with $1 \mathrm{~m} f / 2$ to $1.5 \mathrm{~m} f / 3$ apertures. Here we describe the $1.5 \mathrm{~m} f / 3$ solution.

Y. Kondo (ed.), Observatories in Earth Orbit and Beyond, 487-491.

(C) 1990 Kluwer Academic Publishers. Printed in The Netherlands. 


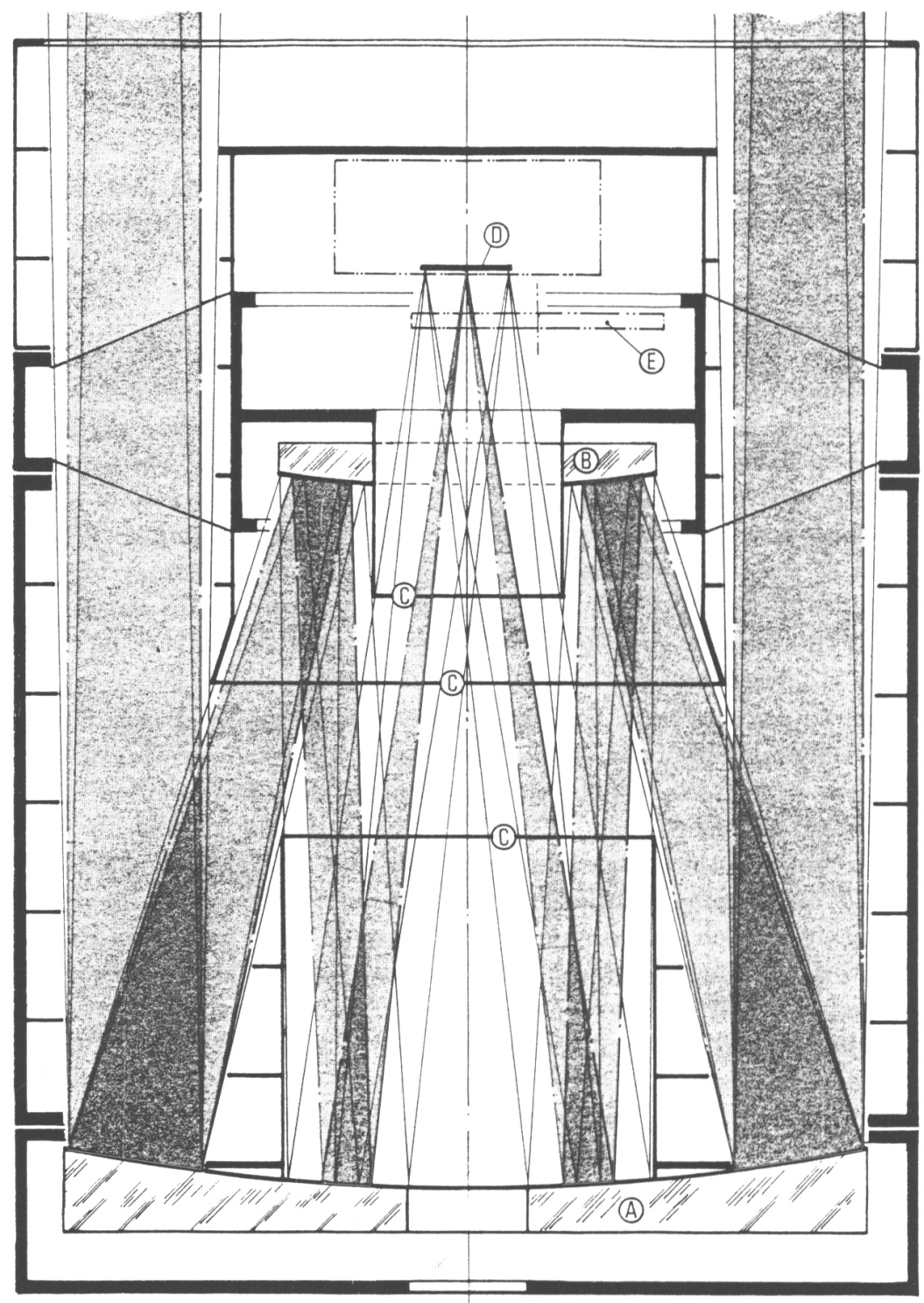

Fig. 1. The schematic layout of the TRUST telescope. A is the primary mirror which also acts as third reflecting surface. $B$ is the secondary mirror. $C$ are the baffles. D is the focal plane, and $E$ is the filter wheel. 


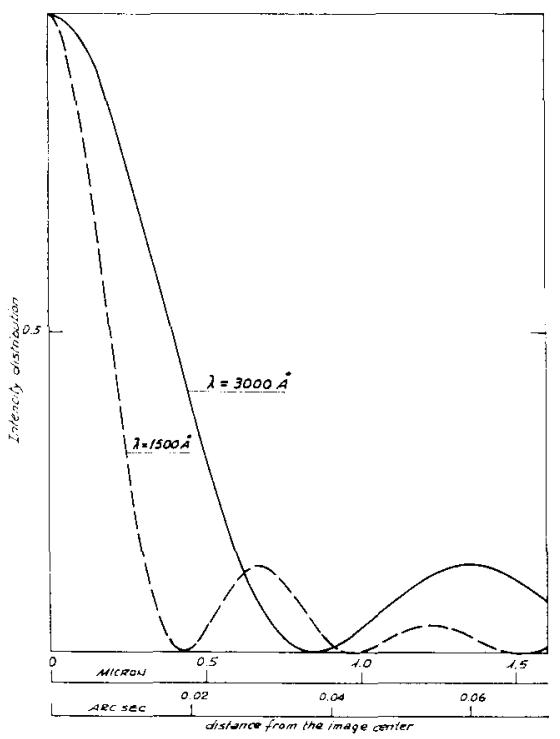

Fig. 2. The point spread function on the axis at the focal plane.

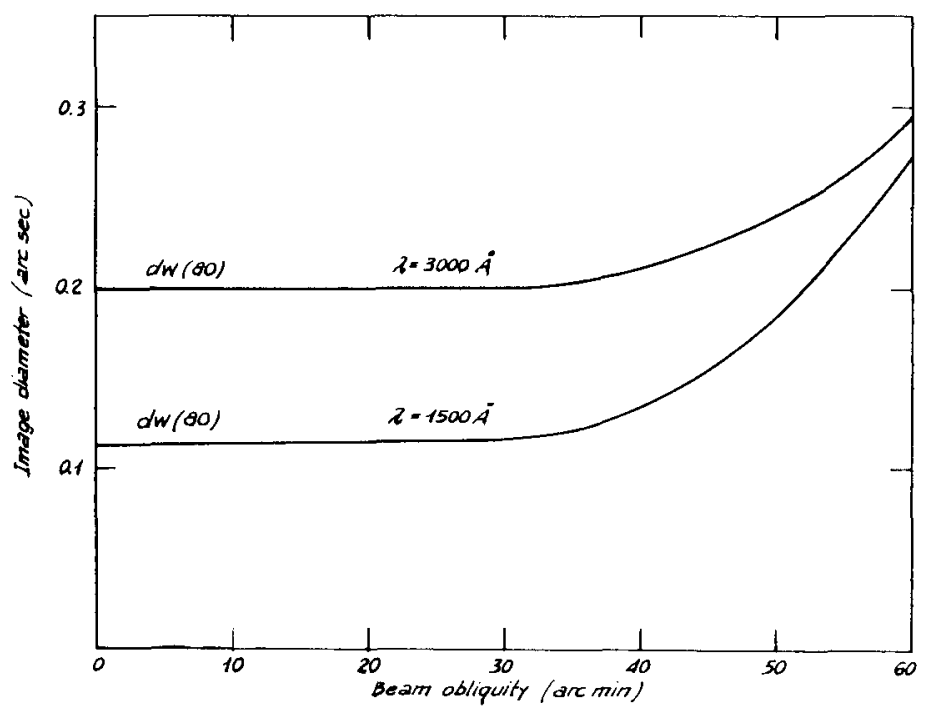

Fig. 3. The diameter of the circumference encircling $80 \%$ of the beam energy at 150 and $300 \mathrm{~nm}$ as a function of the beam obliquity. 

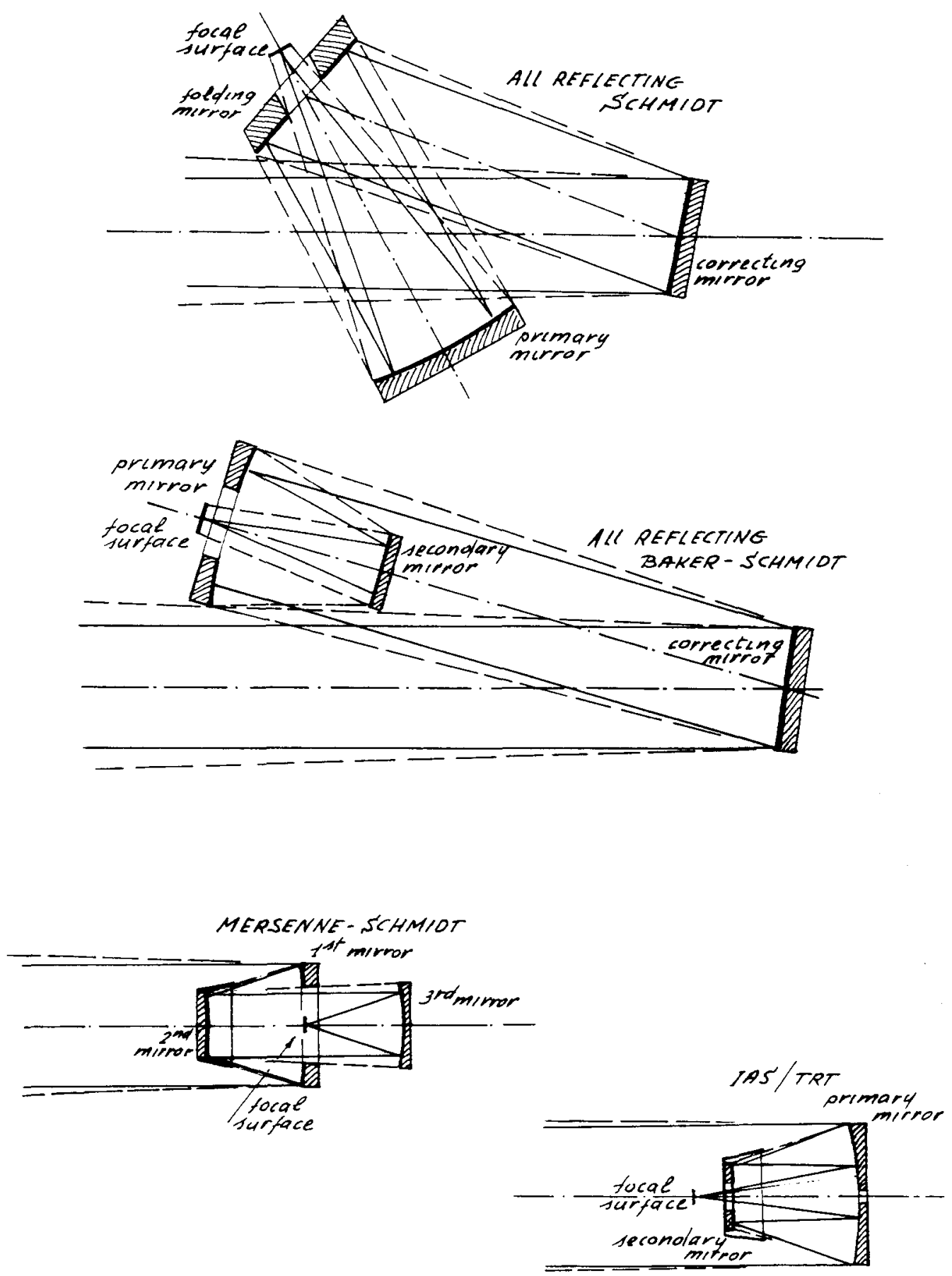

Fig. 4. Comparison of TRUST with other wide-field telescopes. 


\section{The Trust Project}

The TRUST (Three Reflection Ultraviolet Space Telescope) project is based on a $1.5 \mathrm{~m} \mathrm{f} / 3$ three reflection telescope which is shown in Figure 1 . The main characteristics of the instrument is the $f / 1.5$ primary mirror (A) which is acting both as first and as a third reflecting surface. This configuration greatly simplifies the mechanical structure of the telescope, ensures an easier optical alignement and a high compactness of the telescope (see Amoretti et al. 1989). There is also large room for instrumentation near the focal plane (D). High order spherical aberration and coma, as well as third order astigmatism, are corrected by a suitable choise of the figuring parameters, which results in a 2 deg-diameter corrected FOV. Figure 2 shows the on-axis PSF at 150 and $300 \mathrm{~nm}$. The figure clearly shows that, in spite of the large obscuration of the secondary mirror (B), the resolution (FWHM of the central peak) is $0.02-0.04$ arcsec. At $60^{\prime}$ from the axis the resolution is still smaller than $0.2^{\prime \prime}$. Figure 3 gives the dependence of the image size (diameter of the circumference encircling $80 \%$ of the beam energy) on the beam obliquity.

In Figure 4 we finally compare TRUST with other proposed wide-field telescopes. The all-reflecting Schmidt and Baker-Schmidt telescopes (SWAT 1979) are much less compact than TRUST, and the resolution is much worser. Moreover, in the first telescope the focal plane is not flat. Both have a FOV of $4 \mathrm{deg}$, but the field cannot be explored with a good resolution. The Marsenne-Schmidt (Willstrop 1984) has about the same compactness and resolution of TRUST, and a larger FOV, but its configuration is not convenient since the first and third reflecting surfaces are separated, the position of the focal plane is not satisfying, and there is little room for instrumentation. The larger FOV is not a real advantage as it would be difficult to find a detector which would exploit the whole field at full resolution. In conclusion, the TRUST project, for its compactness (hence less weight and smaller on-board encumbrance), wide corrected and unvignetted FOV, high angular resolution, and flat focal surface, appears an ideal economy-class telescope for a space-borne all-sky survey experiment.

\section{References}

Amoretti, M., Badiali, M., and Preite-Martinez, A.: 1989, Astron. Astrophys. 211, 250 SWAT: 1979, Spacelab Wide Angle Telescope, Working Group Report, NASA, Goddard Space Flight Center

Willstrop, R.V.: 1984, Mon. Not. R. Astr. Soc. 210, 597 